\title{
Zonas de catástrofe por eventos hidrometeorológicos en Chile y aportes para un índice de riesgo climático ${ }^{1}$
}

\author{
Cristián Henríquez², Nicolle Aspee ${ }^{3}$ y Jorge Quense ${ }^{4}$
}

\begin{abstract}
RESUMEN
Se estima que el cambio en los patrones globales del clima así como su dinámica natural pueden ocasionar una alta incidencia en los fenómenos extremos y en consecuencia aumentar los niveles de riesgos. En este estudio se revisan los principales desastres climáticos e hidrometeorológicos que han afectado el país entre 19842013, a partir de los decretos que declaran zonas de catástrofe (Ley 16.282) por temporales de lluvia, sequías, heladas, nevazones, inundaciones y movimientos en masa desencadenados por temperaturas y precipitaciones extremas. Por otro lado, información de exposición, vulnerabilidad y resiliencia es integrada en un índice de riesgo climático a nivel comunal. De acuerdo a los resultados obtenidos las comunas del país que presentan mayor riesgo climático son las ubicadas en el litoral de la zona central. Se concluye sobre la necesidad de usar un enfoque adaptativo y no reactivo en el manejo del riesgo especialmente frente a las amenazas que impone el cambio climático.
\end{abstract}

Palabras clave: Eventos climáticos extremos, zonas de catástrofe, índice de riesgo climático.

\begin{abstract}
It is estimated that changes in global weather patterns and natural dynamics can cause a high incidence of extreme events, and therefore increase risk levels. In this study, we review the major climatic and hydrometeorological disasters that have affected Chile between 1984 and 2013, based on ordinances that establish catastrophe zones (Law 16.282) for natural events such as, rainstorms, drought, frost, snowstorms, floods, and mass movements, caused by extreme temperature and rainfall. Information on exposure, vulnerability and resilience is integrated into a climate risk index at the municipal level. Based on the municipal results for Chile, those areas with increased climate risks are located along the central coast. We conclude that it is necessary to consider an adaptive rather than reactive approach to risk management, especially when coping with threats due to climate change.
\end{abstract}

Key words: Extreme climatic events, catastrophe zones, climate risk index

1 Se agradece el financiamiento de los proyectos Fondecyt $N^{\circ} 1130305$ y № 1100657, así como del Centro de Desarrollo Urbano Sustentable (CEDEUS), Conicyt-FONDAP No 15110020, del Centro Nacional de Investigación para la Gestión Integrada de Desastres Naturales (CIGIDEN), Conicyt-FONDAP No 15110017 y al Centro de Cambio Global UC. También se agradece el apoyo Ana Rickmers en la compilación de la base de datos y a Natalia Pino en la cartografía y edición de este documento. Artículo recibido el 3 de mayo de 2015, aceptado el 11 de septiembre de 2015 y corregido el 4 de enero de 2016.

2 Instituto de Geografía, Pontificia Universidad Católica de Chile (Chile). E-mail: cghenriq@uc.cl

3 Instituto de Geografía, Pontificia Universidad Católica de Chile (Chile). E-mail: nvaspee@uc.cl

4 Instituto de Geografía, Pontificia Universidad Católica de Chile (Chile). E-mail: jquense@uc.cl 
A nivel internacional existe un amplio consenso en relación a los impactos del cambio climático sobre el medio humanizado. Se estima que el cambio en los patrones globales del clima así como su dinámica natural pueden ocasionar una alta incidencia en el aumento e intensidad de las amenazas naturales como inundaciones, sequías, incendios, movimientos en masa, olas de calor, entre otros efectos. Si bien el cambio climático se asocia a las alteraciones en las condiciones medias del clima y a la variabilidad de sus propiedades que persisten por un periodo largo de tiempo (IPCC, 2012), cada vez más se asocia a la intensificación de los eventos climáticos extremos. En este sentido, el último informe del Panel Intergubernamental de Cambio Climático (IPCC, 2014) destaca que las ciudades recibirán el mayor impacto relacionado a desastres por fenómenos extremos. Estos incluyen aumento en el nivel del mar, del oleaje por tormentas, de estrés por calor, de precipitaciones extremas, de inundaciones, de sequías, deslizamientos, de aridez y de contaminación del aire (IPCC, 2014).

En Chile, los modelos climáticos regionales estiman para las próximas décadas un aumento de las temperaturas y una disminución de las precipitaciones, especialmente en Chile central, mientras que para el zona norte se pronostica una mayor incertidumbre (Comisión Nacional del Medio Ambiente, 2006). Otras modelaciones orientadas a la proyección de índices climáticos extremos (Villarroel, 2013), tales como temperaturas máximas, mínimas y precipitaciones, reafirman tales tendencias.

La importancia de considerar estos eventos extremos es que cuando estos ocurren producen severas alteraciones en el normal funcionamiento de una sociedad y la comunidad. En situaciones críticas estos episodios pueden desencadenar un desastre o catástrofe, en donde se producen importantes daños humanos, materiales, económicos o ambientales que requieren de una respuesta de emergencia inmediata para satisfacer las necesidades humanas y que pueden requerir ayuda externa para su recuperación (Wilches-Chaux, 1989; IPCC, 2012). En el caso chileno cuando un desastre alcanza niveles superlativos, el Poder Ejecutivo puede declarar zona de catástrofe lo cual permite tomar una serie de medidas y liberar recursos para enfrentar esta situación de crisis en áreas específicas afectadas.

De esta manera podemos entender como riesgo, a una función que conjuga factores de amenaza y vulnerabilidad (Arenas et al., 2010), y que cuando se materializa se transforma en desastre. Esto sucede generalmente cuando se rompe el umbral de tolerancia del funcionamiento normal de un sistema socioecológico (Gallopin, 2006), como por ejemplo cuando una lluvia intensa no puede ser contenida por los sistemas de evacuación respectivos y en consecuencia inunda sectores ocupados por viviendas. Los efectos pueden ser más intensos cuando la población es más vulnerable, por ejemplo cuando afecta a los habitantes de menores recursos económicos, y cuando las condiciones de adaptación y recuperación son débiles, es decir presenta una baja resiliencia. Esta última entendida como la capacidad de los sistemas sociales y económicos para reponerse de la tensión y el impacto de una crisis (Klein et al., 1998; Walker et al., 2002).

La forma más efectiva para hacer frente a estos eventos tanto presentes como futuros es la reducción de riesgo de desastre (RRD), definida como:

“el proceso sistemático de utilizar decisiones administrativas, organizaciones, destrezas y capacidades operativas para ejecutar políticas y fortalecer las capacidades de afrontamiento, con el fin de reducir el impacto adverso de las amenazas naturales $y$ la posibilidad de que ocurra un desastre" (UNISDR, 2009; ECHO, 2012).

En este contexto, el propósito de esta comunicación consiste, por un lado, analizar la distribución de los eventos climáticos extremos, que hayan involucrado la declaración de zona de catástrofe por parte del Estado de Chile, entre los años 1984-2013. Para tal efecto se entenderá como evento climático extremo, de acuerdo a la clasificación de Ayala-Carcedo y Olcina (2002), a fenómenos como granizadas, heladas, Iluvias, neblinas, nevadas, olas de calor, sequías, tempestades, tormentas eléctricas y vendavales. Además se incluyen eventos 
de tipo hidrometeorológicos, correspondientes a crecidas e inundaciones producidas por precipitaciones extremas, fusión de nieve o hielo, desbordamiento de canales, rotura de presas o combinación de varios factores. Por último, se incluyen también eventos como los aluviones y avenidas torrenciales desencadenados por Iluvias intensas.

Por otro lado, interesa además relacionar lo anterior con información de exposición, vulnerabilidad y resiliencia de la población, mediante la propuesta de un índice de riesgo climático, con el objeto de identificar aquellas comunas de Chile con mayor nivel de riesgo climático. Finalmente, es de especial interés proyectar las tendencias de cambio climático sobre el comportamiento de los eventos climáticos extremos que pudiesen afectar al país en los próximos años.

\section{Metodología}

El área de estudio está conformada por 346 comunas del país. Debido a la carencia de datos necesarios para la construcción del índice de riesgo climático, no fueron consideradas las siguientes comunas: Colchane, Ollagüe, Hualañé, Alto Hospicio, Alto del Carmen, Alto Biobío, Cholchol, Hualpén, Isla de Pascua, Juan Fernández, Chaitén Lago Verde, Laguna Blanca, O’Higgins, Guaitecas, Río Verde, Timaukel, Torres del Paine, Tortel, Palena, Primavera, Cabo de Hornos y Antártica.

\section{Evolución de las zonas de catástrofes}

Para determinar la evolución de los eventos climáticos extremos, se generó una base de datos a través de la revisión retrospectiva de los decretos de declaración de zonas de catástrofe entre los años 1984 y 2013, promulgados por el Ministerio del Interior según el Decreto Supremo No 104, del 25 de junio de 1977, que coordina y sistematiza el Título I de la Ley $N^{\circ} 16.282$ (1965), sobre disposiciones permanentes para casos de sismos o catástrofes y sus modificaciones posteriores (1986, 1991, 2003, 2010 y 2012). Para ello se consultó la base de datos de leyes de la Biblioteca del Congreso Nacional (BCN, 2014) de Chile, para luego sistematizar la información por región, comuna, fecha, tipo de evento (lluvias, sequías, heladas, nevadas y aluviones) y observaciones principales del evento. Este periodo de análisis responde a la disponibilidad de los decretos catastrados.

\section{Determinación del índice de riesgo climático}

Una vez sistematizada la información de catástrofes climáticas, se propone un índice de riesgo climático a escala comunal, que integra indicadores de amenaza, exposición, vulnerabilidad y resiliencia, usando variables proxy de riesgo, mediante la siguiente fórmula:

$$
R_{\mathrm{i}}=\frac{\mathrm{A}+((\mathrm{E}+\mathrm{V}+\mathrm{Re}) / 3)}{2}
$$

Donde:

$R_{i}$ : índice de riesgo climático

$A$ : indicador normalizado de amenaza

E: indicador normalizado de exposición

$V$ : indicador normalizado de vulnerabilidad

Re: indicador normalizado de resiliencia

En primer lugar, la amenaza fue calculada a través de la frecuencia de zonas de catástrofe declaradas por comuna, entre 1984 y 2013, de acuerdo al paso descrito en la fase anterior. Estos eventos son considerados como el peor escenario climático, de acuerdo a su presencia y extensión espacial.

La exposición fue calculada mediante el porcentaje de población infantil (0 a 15 años) y adulta mayor (sobre 65 años) a nivel comunal, extraídas del Censo de Población y Vivienda año 2002 del Instituto Nacional de Estadística (INE). El supuesto es que esta población se encuentra más expuesta ante un evento extremo de origen climático que la población adulta (IPCC, 2014). Es importante aclarar que no se usaron los datos del censo de población y vivienda de 2012, ya que estos se encuentran objetados por graves deficiencias en el levantamiento de datos.

La vulnerabilidad se ha abordado a través de las características socioeconómicas y de salud de la población, analizando el porcentaje de población en situación de pobreza y el 
porcentaje de población con una o más enfermedades respiratorias a nivel comunal. Para el cálculo de las enfermedades respiratorias, se utilizaron las respuestas positivas de las personas que declararon haber estado en tratamiento por alguna de las siguientes enfermedades: neumonía, asma, enfermedad pulmonar obstructiva crónica (EPOC) o infección respiratoria aguda, de acuerdo a la información proporcionada por el Departamento de Estadísticas e Información de Salud del Ministerio de Salud (DEIS, 2012). Se trabaja sobre el supuesto que estas enfermedades se manifiestan con mayor frecuencia después de un evento extremo, como heladas o lluvias intensas (D'Amato et al., 2012).

Para el caso de la variable de pobreza, se usó información de la encuesta CASEN, a partir del documento "Incidencia de la Pobreza a nivel Comunal, según Metodología de Estimación para Áreas Pequeñas", que incluye estimaciones de pobreza a nivel comunal que corrigen el sesgo muestral a ese nivel (Ministerio de Desarrollo Social, 2013).

Finalmente, el indicador de resiliencia se ha estimado usando el valor del índice de desarrollo humano (IDH), año 2003, a nivel comunal de acuerdo a la información del Programa de Naciones Unidas para el Desarrollo (MIDEPLAN y PNUD, 2005) y el porcentaje de población perteneciente a organizaciones comunitarias a través de la base de datos de la encuesta CASEN año 2009 (MIDEPLAN, 2009). El primer índice se ha escogido en función de determinar aquellas comunas que tienen mejores condiciones de ingreso, salud y educación para enfrentar una crisis. Mientras que el segundo se asocia a identificar comunas donde hay comunidades con mayor nivel de participación social, en instituciones como juntas de vecinos, clubes deportivos y otras organizaciones sociales, que en principio pueden enfrentar de mejor manera un desastre natural y también recuperarse después de una perturbación.

Una vez levantada la información base se procedió a una fase de normalización, para lo cual se usó el método de designación de unidades valorativas propuesto por Carvacho (2011). Este método permite transformar los atributos de las variables medidas en unidades heterogéneas en unidades homogéneas, en una escala de 0 a 1 , donde 0 corresponde al mínimo valor de la variable y 1 al máximo, considerando una fórmula que aplica dos funciones de asignación de puntaje; una para los puntajes sobre el promedio y otra para los puntajes bajo el promedio, debido a que no necesariamente los recorridos de los puntajes a ambos lados de la media son simétricos (Carvacho, 2011). La fórmula utilizada corresponde a:

$$
\begin{gathered}
P_{i}=\left\{\begin{array}{c}
X_{\mathrm{i}} \geq \bar{x} \rightarrow P_{\text {med }}+\left(X_{\mathrm{i}}-\bar{x}\right) \times R_{\text {sup }} \\
X_{\mathrm{i}}<\bar{x} \rightarrow P_{\text {min }}+\left(X_{\mathrm{i}}-x_{\text {min }}\right) \times R_{\text {inf }}
\end{array}\right. \\
R_{\text {sup }}=\frac{P_{\text {max }}-P_{\text {med }}}{X_{\text {max }}-\bar{x}} \\
R_{\text {inf }}=\frac{P_{\text {max }}-P_{\text {med }}}{\bar{x}-X_{\text {min }}}
\end{gathered}
$$

Donde:

$P_{i}$ : puntaje normalizado

$X_{i}$ : valor de la observación $i$ de la variable

$X$ : promedio de la variable original

$X_{\min }$ : valor mínimo de la variable

$X_{\max }$ : valor máximo de la variable

$R_{\text {sup }}$ : razón de puntaje que se asigna a cada unidad de $X$ sobre el promedio

$R_{\text {inf }}$ : razón de puntaje que se asigna a cada unidad de $X$ bajo el promedio

$P_{\text {med }}$ : valor del puntaje asignado al promedio

$P_{\min }:$ valor del puntaje asignado al valor mínimo de la variable

$P_{\max }$ : valor del puntaje asignado al valor máximo de la variable

Para el proceso de normalización se usaron como parámetros de transformación las medias, mínimas y máximas nacionales de cada variable. Para la pobreza y enfermedades respiratorias se usó como unidad de medida los porcentajes de la variable en relación al total comunal. Para el caso de la población expuesta se usó un criterio distinto de normalización, ya que se empleó como valor de entrada el porcentaje de la población infantil y adulta de cada comuna en relación al total nacional: en este caso las comunas que tienen mayor cantidad bruta de población en relación al país son las más expuestas. En el caso del IDH y población que participa en organizaciones comunitarias se ha invertido el sentido de los puntajes, de modo que las comunas con mayores porcentajes representen el mejor nivel de resiliencia (valores cercano a 0) y los porcentajes más bajos la peor situación de resiliencia (valores cercano a 1). El Cuadro $\mathrm{N}^{0} 1$ muestra los valores de referencia para la normalización de cada variable. 
Cuadro $\mathrm{N}^{\mathrm{o}} 1$

Valores de referencia para la normalización de las variables a nivel comunal

\begin{tabular}{|c|c|c|c|c|c|}
\hline Variables & $\begin{array}{l}\text { Valor } \\
\text { mínimo }\end{array}$ & $\begin{array}{l}\text { Valor } \\
\text { máximo }\end{array}$ & $\begin{array}{l}\text { Valor } \\
\text { promedio }\end{array}$ & Fuente & $\begin{array}{l}\text { Indica- } \\
\text { dor }\end{array}$ \\
\hline $\begin{array}{l}\text { Zonas de catástrofe (frecuen- } \\
\text { cia) }\end{array}$ & 0 & 9 & 3,3 & $\begin{array}{c}\mathrm{BCN} \\
(2014)\end{array}$ & $\begin{array}{l}\text { Amena- } \\
\text { za }\end{array}$ \\
\hline $\begin{array}{l}\text { Población infantil y adulto } \\
\text { mayor expuesta }(\%)\end{array}$ & 0 & 3,24 & 0,3 & INE (2002) & $\begin{array}{l}\text { Ex- } \\
\text { posición }\end{array}$ \\
\hline Pobreza (\%) & 0 & 100 & 16,9 & $\begin{array}{c}\text { Ministerio de } \\
\text { Desarrollo Social } \\
(2013)\end{array}$ & \multirow[t]{2}{*}{$\begin{array}{l}\text { Vulnera- } \\
\text { bilidad }\end{array}$} \\
\hline Enfermedades respiratorias (\%) & 0,27 & 4,95 & 1,94 & DEIS (2012) & \\
\hline $\begin{array}{l}\text { Índice de desarrollo humano } \\
\text { (IDH) }\end{array}$ & 0 & 1 & 0,725 & $\begin{array}{l}\text { MIDEPLAN y } \\
\text { PNUD (2005) }\end{array}$ & \multirow{2}{*}{$\begin{array}{l}\text { Resilien- } \\
\text { cia }\end{array}$} \\
\hline Participación comunitaria (\%) & 0 & 100 & 25,2 & $\begin{array}{l}\text { MIDEPLAN } \\
(2009)\end{array}$ & \\
\hline
\end{tabular}

Fuente: Elaboración propia.

En los casos de los indicadores compuestos por dos variables, como vulnerabilidad y resiliencia, fueron integrados, previa normalización, usando igual peso entre las variables.

\section{Proyección del cambio climático y la exposición}

Finalmente, para estimar las tendencias de cambio climático y exposición, se han usado las modelaciones de precipitaciones y temperaturas para los años 2010-2100 desarrolladas por el Departamento de Geofísica de la Facultad de Ciencias Físicas y Matemáticas de la Universidad de Chile (DGF/CONAMA, 2006) y las proyecciones de población del Instituto Nacional de Estadística (INE, 2005) para el año 2020. Los resultados se presentan para las cinco macrozonas del país: Norte Grande, Norte Chico, Zona Central, Zona Sur y Zona Austral.

\section{Evolución de desastres y catástrofes en Chile}

La determinación de zonas afectadas por eventos extremos obedece a las disposiciones establecidas en la Constitución Política de la República de Chile (1980) y en particular a la Ley Orgánica Constitucional de los Estados de Excepción Constitucional, Ley $N^{\circ} 18.415$ (1985), en donde se establecen una serie de normas particulares para cada situación. Los estados de excepción, según el artículo 39 de la Constitución, corresponden a situaciones en las que ciertos eventos afecten gravemente el normal funcionamiento del Estado, tales como guerras, conmoción interior, emergencia y calamidad pública.

A través de la declaración de un estado de excepción, dependiendo de sus características, puede verse afectado el ejercicio de los derechos y garantías de las personas, con el fin de su protección. Estos estados pueden ser "Estado de asamblea" en caso de guerra exterior, "Estado de sitio" en caso de guerra interior, "Estado de emergencia" en caso de grave alteración del orden público, daño o peligro para la seguridad de la Nación por motivos internos o externos, y finalmente "Estado de catástrofe" para situaciones de calamidad pública.

Como se ha mencionado anteriormente, en el caso de zonas afectadas por eventos naturales estas pueden ser decretadas como zonas de catástrofe a través de un Decreto Supremo promulgado por el Ministerio del Interior, según lo dispuesto en la Constitución y la 
Ley № 16.282 (1974). Además las medidas que se adopten en cada declaración dependerán de la Ley de Presupuestos para el sector público según el período que corresponda. A partir de la declaración de zona de desastre, la normativa establece excepciones en cuanto a cambios en los presupuestos públicos para facilitar la gestión de daños y de ayuda a damnificados, en este sentido se establece un marco para generar préstamos con garantías especiales, recibir y canalizar donaciones con facilidades tales como exención de impuestos y tarifas de carga o descarga, movilización, almacenaje entre otros cargos, con el fin de agilizar la ayuda recibida.

En términos administrativos, el Presidente de la República y los encargados de los organismos públicos designados por el Presidente se deben reunir en la Oficina Nacional de Emergencias del Ministerio del Interior y Seguridad Pública (ONEMI) para constituir el Comité de Emergencia (COE) y evaluar los daños y determinar las medidas a seguir (ONEMI, 2013).

Respecto a la forma de operar de las zonas de catástrofe, se puede señalar el evento de junio del año 2000 donde cinco sistemas frontales y un núcleo frío en altura produjeron anegamientos, crecidas de ríos e inundaciones, que resultaron en graves daños a viviendas, servicios y muerte de personas, desencadenando una declaratoria de zona de catástrofe para las comunas de Loncoche, Gorbea, Valdivia, San José de La Mariquina, La Unión y Panguipulli, mediante Decreto Supremo 3.094 del 5 de junio, firmado por el Presidente de la República en su visita a la zona. Posteriormente, con fecha 12 de junio, mediante Decreto Supremo No 3.111, se amplía la zona a las comunas de Los Lagos, Lanco y San Pablo. El miércoles 14 de junio, el Presidente se constituyó en la ONEMI, junto al Ministro de Obras Públicas y Telecomunicaciones, los Subsecretarios del Interior, Obras Públicas y Educación, Intendentes Metropolitanos, Director y técnicos de ONEMI, para evaluar la emergencia producto de los temporales. Además, se decretó mediante el Decreto Supremo (D.S.) № 3.120 zonas afectadas por catástrofe en las regiones de Valparaíso y Metropolitana y se determinó la suspensión de clases para los niveles prebásico, básico y medio de establecimientos públicos y privados de estas regiones más las regiones VI y VII (ONEMI, 2000).
En este caso la gestión de la ONEMI se centró en funciones de captura y administración de información sobre daños, entrega de información oficial a autoridades y prensa, apoyo en terreno de un equipo de profesionales, canalización de la ayuda del Gobierno hacia las regiones y zonas afectadas por los temporales y labores de coordinación en general. Dentro de la ayuda realizada se puede mencionar la entrega de un total de 8.620 cajas de alimentos, 9.615 colchonetas, 18.794 frazadas, 5.500 planchas de zinc, 13.350 planchas de pizarreño, 22.275 pañales desechables y 1.870 viviendas de emergencia, entre otras (ONEMI, 2000).

Por último, es importante mencionar que luego del terremoto del 2010 y como parte de una de las recomendaciones de la misión de las Naciones Unidad que visitó el país para dar cumplimiento al Marco de Acción de Hyogo, firmado por Chile el 2005, se constituyó una plataforma nacional para la reducción del riesgo de desastres, liderada por la ONEMI. Uno de los resultados de este trabajo es la Política Nacional para la Gestión del Riesgo de Desastres (ONEMI, 2014). Esta política nacional define un marco de acción para cinco ejes estratégicos: fortalecimiento institucional, fortalecimiento de los sistemas de monitoreo y alerta temprana, fomento de la cultura de la prevención y autoaseguramiento, reducción de los factores subyacentes del riesgo y fortalecimiento de la preparación ante los desastres.

\section{Propuesta de un índice de riesgo climático}

En este apartado se presenta la propuesta de índice de riesgo climático, compuesta por los indicadores parciales de amenaza, exposición, vulnerabilidad y resilencia.

\section{Zonas de catástrofes en Chile como indicador de amenaza}

En función de la revisión de los decretos se han registrado 78 eventos asociados a zonas de catástrofes y desastres en el periodo comprendido desde el año 1984 al 2013 (Figura $N^{0} 1$ ). La macrozona más afectada ha 
sido la Zona Central del país con el 38,5\% de los eventos, seguida de la Zona Sur, con $32,1 \%$, Norte Chico 18\%, Norte Grande 7,7\% y Austral con 3,9\%. Las comunas que presentan mayor frecuencia son: La Ligua, Cabildo y Petorca con nueve eventos catastróficos.

Figura $N^{\circ} 1$

Evolución de zonas de catástrofes por causas climáticas e hidrometeorológicas en Chile 19842013

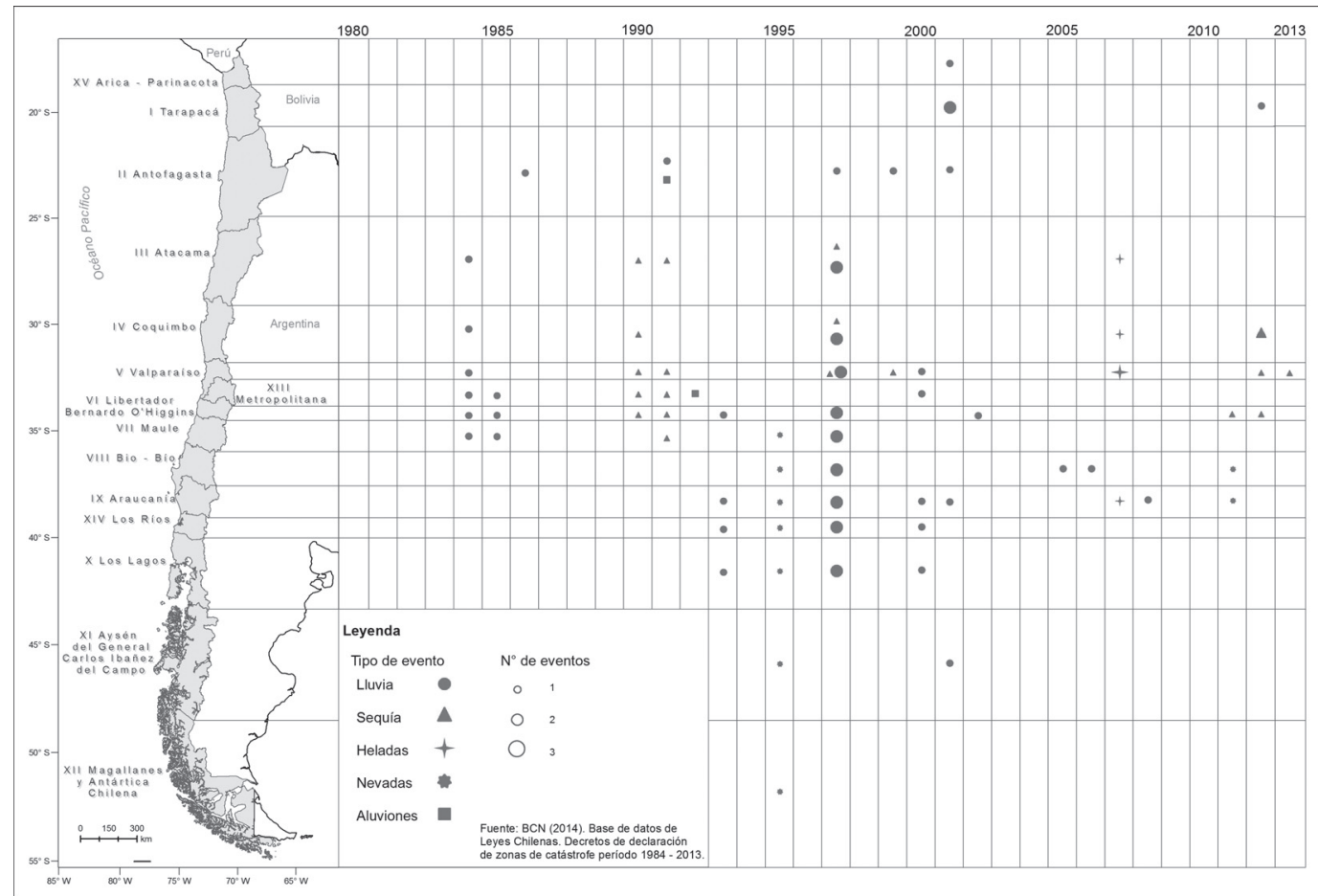

Fuente: Elaboración propia en base a datos recolectados de www.leychile.cl (BCN, 2014).

Con respecto a la naturaleza de estos eventos, los temporales de lluvia han sido el tipo de evento más recurrente con un $61 \%$, siendo el año 1997 el más importante, ya que concentró la mayor cantidad de eventos afectando 10 regiones.

La segunda tipología de evento más recurrente son las sequías con un $20 \%$ para el periodo, seguida de las nevazones con un $9 \%$, las heladas con un $7 \%$ y finalmente los aluviones con solo 3 eventos. Estos últimos, constituyen eventos extremos puntuales desencadenados por las lluvias intensas, los más importantes se han localizado en la región de Norte Grande, en la ciudad de Antofagasta en el año 1991 (D.S. 513), comunas de Camiña y Huara (D.S. 289) en 2012, y en la comuna de La Florida el año 1993 (D.S. 765).

Es interesante notar que la tendencia es hacia un aumento en el número de eventos climáticos extremos con un peak el año 2007, año que se caracterizó por la concurrencia de nevadas y heladas en las macrozonas del Norte Chico y Central.

\section{Indicador de exposición}

Respecto al segundo componente del índice, existen 5.100 .458 personas que están expuestas a una amenaza climática (población menor a 15 
años y mayor a 65 años), equivalente a 33,8\% de la población del país. Estas se concentran preferentemente en el Área Metropolitana de Santiago (AMS) en la macrozona Central. Las comunas más expuestas son Puente Alto, con un $3,2 \%$ de la población expuesta, le siguen las comunas de Maipú y La Florida con un 2,83\% y un 2,06\% respectivamente. Otras comunas del AMS que presentan alto nivel de exposición son San Bernardo, Las Condes y Peñalolén. Es interesante señalar que las comunas que se encuentran en el piedemonte de la cordillera de los Andes son mayormente afectadas por eventos climáticos extremos, tales como deslizamientos de terreno y aluviones, por lo que su alto nivel de exposición constituye un punto clave en la gestión del riesgo.

Otras comunas con un alto nivel de exposición en la macrozona Central del país son Quintero, Valparaíso y Rancagua. En el Norte Grande figuran las comunas de Antofagasta y Arica y en la Zona Sur las comunas mayormente expuestas son Temuco y Puerto Montt (Figura No 2).

Figura $\mathrm{N}^{\circ} 2$

Mapa de exposición ante amenazas climáticas 2002

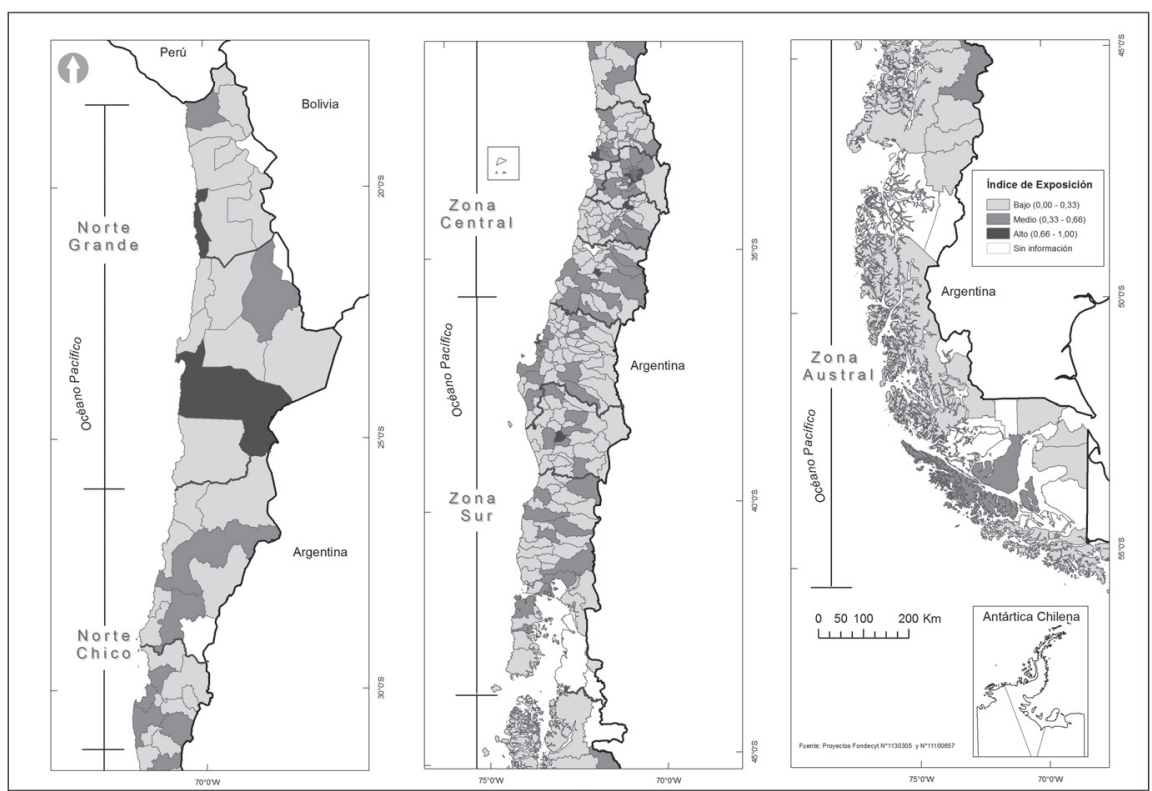

Fuente: Elaboración propia en base a datos Censo de Población y Vivienda (INE, 2002).

\section{Indicador de vulnerabilidad}

Como se mencionó en la metodología, este indicador se compone en dos variables, la pobreza y las enfermedades respiratorias. En el primer caso, Chile ha mejorado sus indicadores sociales, pasando el año 1990 de $38,4 \%$ de su población en situación de pobreza a 16,9\% de pobres el 2009 (Ministerio de Desarrollo Social, 2012; 2013). De todas formas el porcentaje de pobreza es superior al promedio de países OCDE que alcanza a 11,3\% al año 2010 (OCDE, 2014).

De acuerdo a los datos de la encuesta CASEN 2009 las comunas más pobres son: Angol (37,6\%), Lumaco (36,8\%), Loncoche $(36,5 \%)$ y Purén $(36,4 \%)$, mientras que cifras más recientes de la encuesta CASEN 2011 señalan un cambio en la posición: Ercilla (48,8\%), Los Álamos (41,3\%), Puerto Saavedra $(37,3 \%)$ y Lonquimay $(36,1 \%)$. En ambos 
registros se observa una concentración de pobreza en macrozona Sur, especialmente en la Región de la Araucanía.

Por su parte, al año 2009 el país presenta una morbilidad promedio asociada a enfermedades respiratorias de 1,9\%. Las comunas que presentan mayores valores corresponden a Lampa $(4,9 \%)$, Chiguayante $(4,7 \%)$ y San Ramón $(4,5 \%)$. Al comparar estos datos con la tasa de egresos hospitalarios por enfermedades respiratorias (DEIS, 2012), se observa que las regiones que concentran mayor can- tidad de enfermedades son las regiones de Araucanía y Los Ríos.

Al integrar estas dos variables se observa que las comunas con mayor indicador de vulnerabilidad son San Ramón, Chiguayante y Lampa, con un valor de 0,74. Estas comunas presentan altos valores de vulnerabilidad explicado por los altos valores las variables parciales; San Ramón posee un $25,8 \%$ de población pobre y $4,5 \%$ de enfermedades, Chiguayante $18,4 \%$ y $4,7 \%$, y Lampa un $16,1 \%$ y $4,9 \%$ respectivamente. La distribución espacial se presenta en la Figura $N^{\circ} 3$.

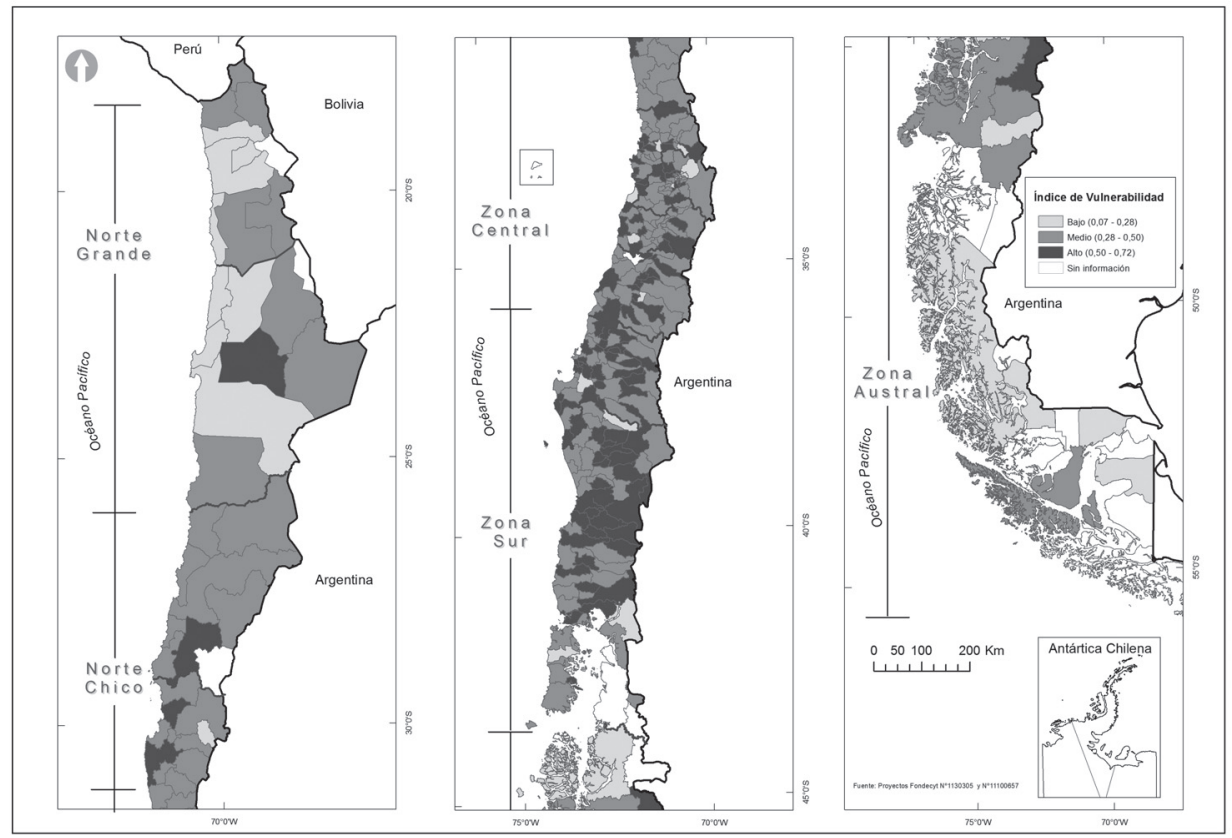

Fuente: Elaboración propia en base a datos del Ministerio de Desarrollo Social (2013) y DEIS (2012).

\section{Indicador de resiliencia}

El IDH, primer componente del indicador de resilencia, muestra que las comunas con mayor desarrollo humano son Vitacura $(0,949)$, Las Condes $(0,933)$ y Lo Barnechea $(0,912)$; mientras que las comunas con menor valor son: San Juan de La Costa $(0,510)$, Treguaco $(0,562)$ y Ninhue $(0,569)$. En segundo término, las comunas con mayor porcentaje de población perteneciente a organizaciones comunitarias son Alto del Carmen $(60,4 \%)$, Currarehue $(56,2 \%)$ y Camiña (54,6\%); y las comunas con menor porcentaje son: Paredones (4,8\%), Yerbas Buenas $(7,5 \%)$ y San Ramón (8,4\%).

De la combinación de ambas variables se obtiene que las comunas más resilientes del país resultaron: Vitacura, con un índice 
de 0,295 , seguida de Camarones $(0,38)$ en el Norte Grande y San Gregorio $(0,39)$ en la macrozona Austral. Llama la atención que entre las comunas con mejores índices se encuentran comunas del extremo norte y sur del país, lo que se puede explicar por el alto nivel de participación en organizaciones sociales ( $52 \%$ y $32,5 \%$, respectivamente), ya que estos valores son muy superiores a la media nacional $(25,2 \%)$.
Las comunas que presentan peores niveles de resiliencia pertenecen a la Zona CentroSur del país: Paredones $(0,72)$, Yerbas Buenas $(0,68)$ y Colina $(0,66)$, en general estas comunas presentan bajos niveles de participación en organizaciones sociales y un bajo índice de desarrollo humano (Figura $N^{\circ} 4$ ).

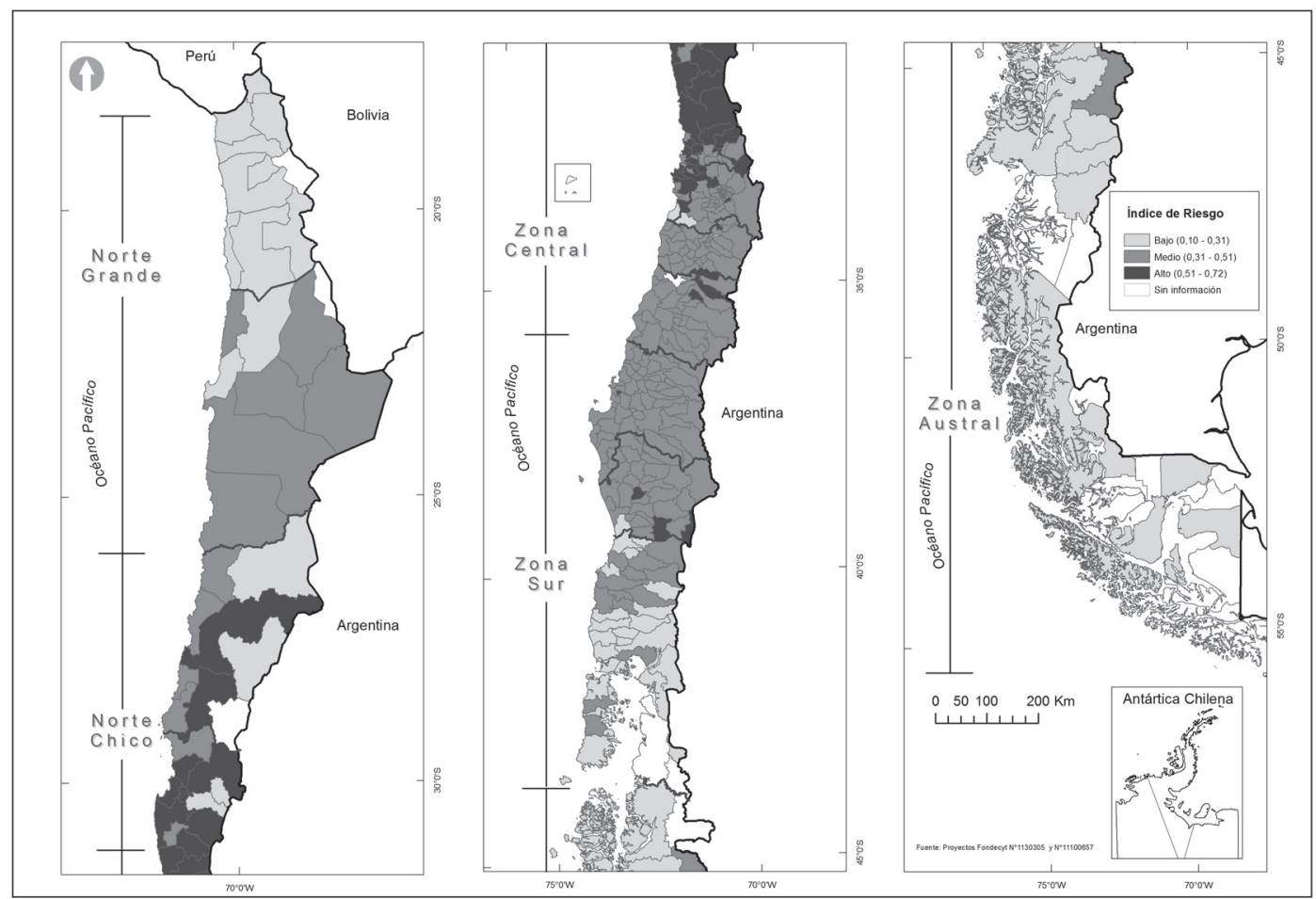

Fuente: Elaboración propia en base a datos de MIDEPLAN y PNUD (2005) y MIDEPLAN (2009).

\section{Índice de riesgo climático en Chile}

Finalmente, una vez normalizados los datos usados en los indicadores, se procedió a integrarlos en el índice de riesgo climático. De esta forma, las zonas del país que presentaron mayores niveles de riesgo climático son el área costera de la macrozona Central y del Norte Chico, en donde se presentan la mayor cantidad de comunas con un índice elevado de riesgo climático. Las comunas con mayores índices de riesgo climático son La Ligua $(0,73)$, Cabildo $(0,69)$ y Petorca $(0,69)$, de la provincia de Petorca, V Región de Valparaíso. Estas comunas se caracterizan por presentar altos niveles de amenaza, con valores sobre la media nacional (3,3 eventos).

Estas comunas registran nueve eventos climáticos extremos asociados a cuatro 
sequías, tres temporales y dos heladas. El primer evento corresponde a los temporales de lluvia que afectaron a gran parte del país el año 1984, estos temporales generaron aludes de nieve, inundaciones, irrupción de caminos y vías férreas, suspensión de servicios públicos, daños a propiedad pública y privada, y muerte de personas. Con motivo de este evento se declaró zona de catástrofe a las regiones Norte Chico y Central (D.S. 741).

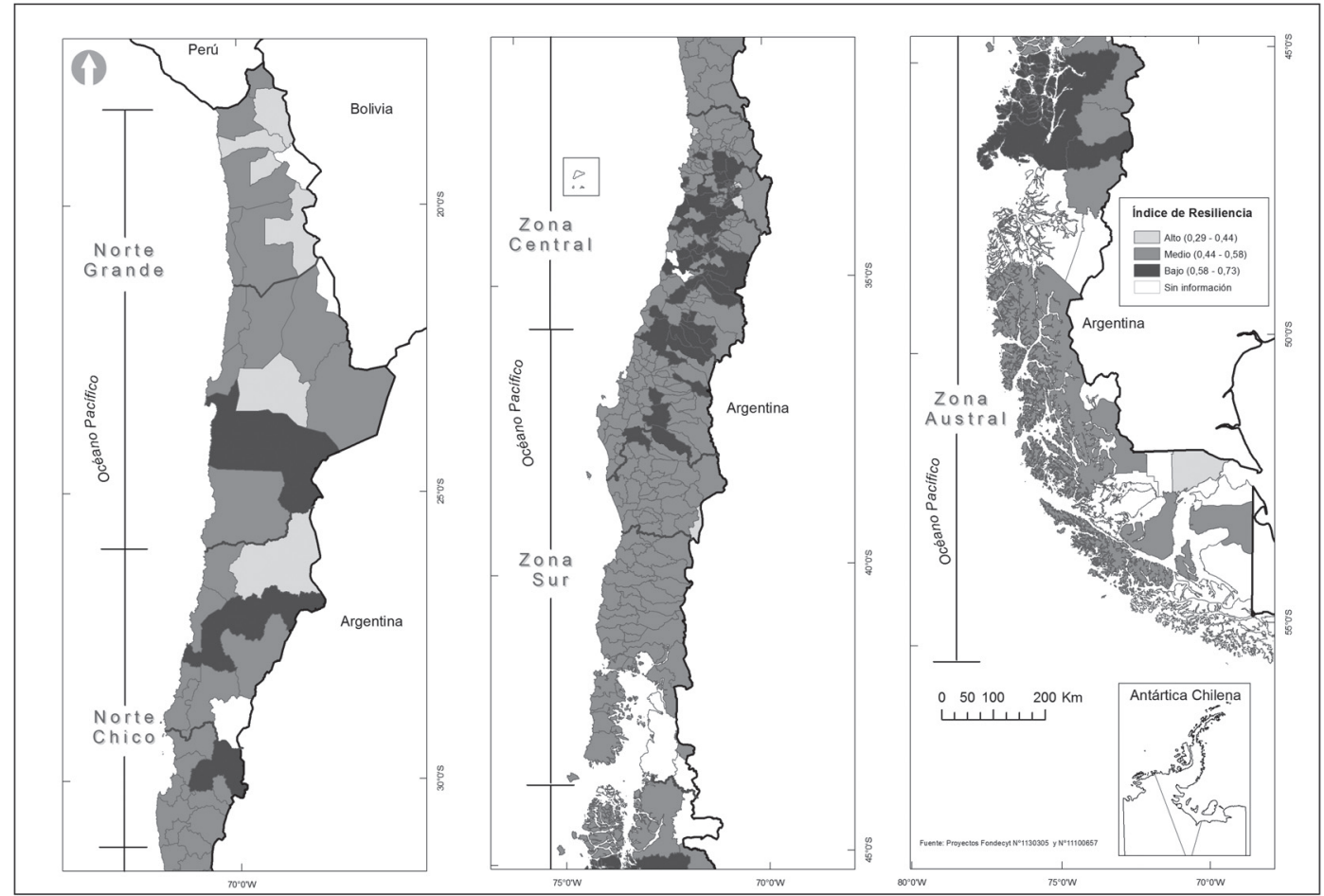

Fuente: Elaboración propia.

Posteriormente, en 1990, una prolongada sequía (D.S. 750) afectó al país con especial intensidad en algunas comunas de las regiones administrativas de Atacama, de Coquimbo y de Valparaíso (parte sur del Norte Chico y zona costera de la macrozona Central), encontrándose Cabildo, La Ligua, Petorca y Zapallar como las áreas más afectadas dentro de esta última región. Entre los efectos de este fenómeno se encontraron grandes pérdidas en las ramas económicas de agricultura, ganadería, minería, y también afectación en cuanto a la disponibilidad de alimentos y de agua.

En 1997 se registraron tres eventos que afectaron a estas comunas; una sequía en marzo que afectó a comunas de las regiones de Atacama, Coquimbo y Valparaíso (D.S. 866), produciendo daños en la agricultura, ganadería y disponibilidad de agua para el riego y consumo humano. En este decreto se excluyen sectores urbanos y luego se incorporan nuevas comunas afectadas por la 
catástrofe, mediante D. S. 1.251. En junio del mismo año fuertes temporales de viento y lluvia afectaron gran parte del país con daños considerables a la población y viviendas, e infraestructura vial, social, urbana y rural (D.S. 1543). A fines de agosto de 1997 se declara zona de catástrofe (D.S. 849) por bajas temperaturas que provocaron la destrucción de cultivos agrícolas, afectando a pequeños agricultores y a empresas, disminuyendo el empleo. Al mes siguiente ocurrieron nuevos temporales con similares efectos a los del evento de junio (D.S. 2.149).

El ciclo de sequías y temporales de Iluvia que afectó a la zona central del país continuó en 1999 con una sequía que afectó gravemente la agricultura, ganadería y disponibilidad de agua declarándose como zona de catástrofe la provincia de Petorca y sus comunas integrantes (D.S. 3.144). El año 2000 se caracterizó por presentar intensas lluvias que provocaron el anegamiento de vías de circulación y paralización del transporte, además de daños en infraestructura pública y privada, en junio de ese año se declaró como zona de catástrofe a las regiones de Valparaíso y Metropolitana de Santiago (D.S. 3.120).

En agosto de 2007 (D.S. 849) fuertes heladas impactaron las comunas de Cabildo, La Ligua y Petorca, las que destruyeron los cultivos de la zona, afectando tanto a pequeños agricultores como a empresarios agrícolas. Finalmente, el evento más reciente es del año 2012 (D.S. 234), en el cual una intensa y prolongada sequía, afectó los sectores agrícola, productivo, ganadero, de la pequeña minería, de riego y de energía eléctrica debido a la reducción del caudal en las cuencas de los ríos Petorca, La Ligua y Aconcagua, afectando nuevamente a las tres comunas con mayor índice de riesgo anteriormente señaladas.

Por otro lado, estas comunas presentan niveles de resiliencia cercanos a la media nacional: La Ligua 0,51, Cabildo 0,5 y Petorca 0,47 . En cuanto a los niveles de exposición, estas comunas poseen cifras menores a la media nacional $(0,29)$ con excepción de la comuna de La Ligua $(0,35)$. Finalmente, respecto a vulnerabilidad Petorca $(0,57)$, La Ligua $(0,49)$ y Cabildo $(0,44)$ son las comunas que se encuentran más vulnerables dentro de la provincia, junto con Papudo $(0,49)$, mientras que Zapallar alcanza el mejor nivel $(0,39)$.

Finalmente, las comunas que presentan menor nivel de riesgo son María Elena, Quinchao, y Puqueldón, con valores bajo 0,15. Si bien todos los indicadores son bajos, el común denominador es que son comunas donde no se ha registrado eventos climáticos catastróficos.

\section{Proyección de variables climáticas y exposición ante el cambio climático}

A partir de las modelaciones realizadas por el modelo PRECIS (Comisión Nacional del Medio Ambiente, 2006), para los próximos años (2010-2040, 2040-2070 y 2070-2100), se proyecta que en el país existirá un aumento de las temperaturas y una disminución de las precipitaciones (Figura $\mathrm{N}^{\circ}$ 6). Una excepción a esta proyección corresponde al comportamiento del Norte Grande donde no hay una tendencia clara, en términos que se podría esperar una disminución de temperaturas en la zona costera y un aumento de precipitaciones en el sector altiplánico (Falvey \& Garreud, 2009). 
Figura $N^{\circ} 6$

Proyección del cambio climático para el período 2010-2100 y estimación de población para el año 2020

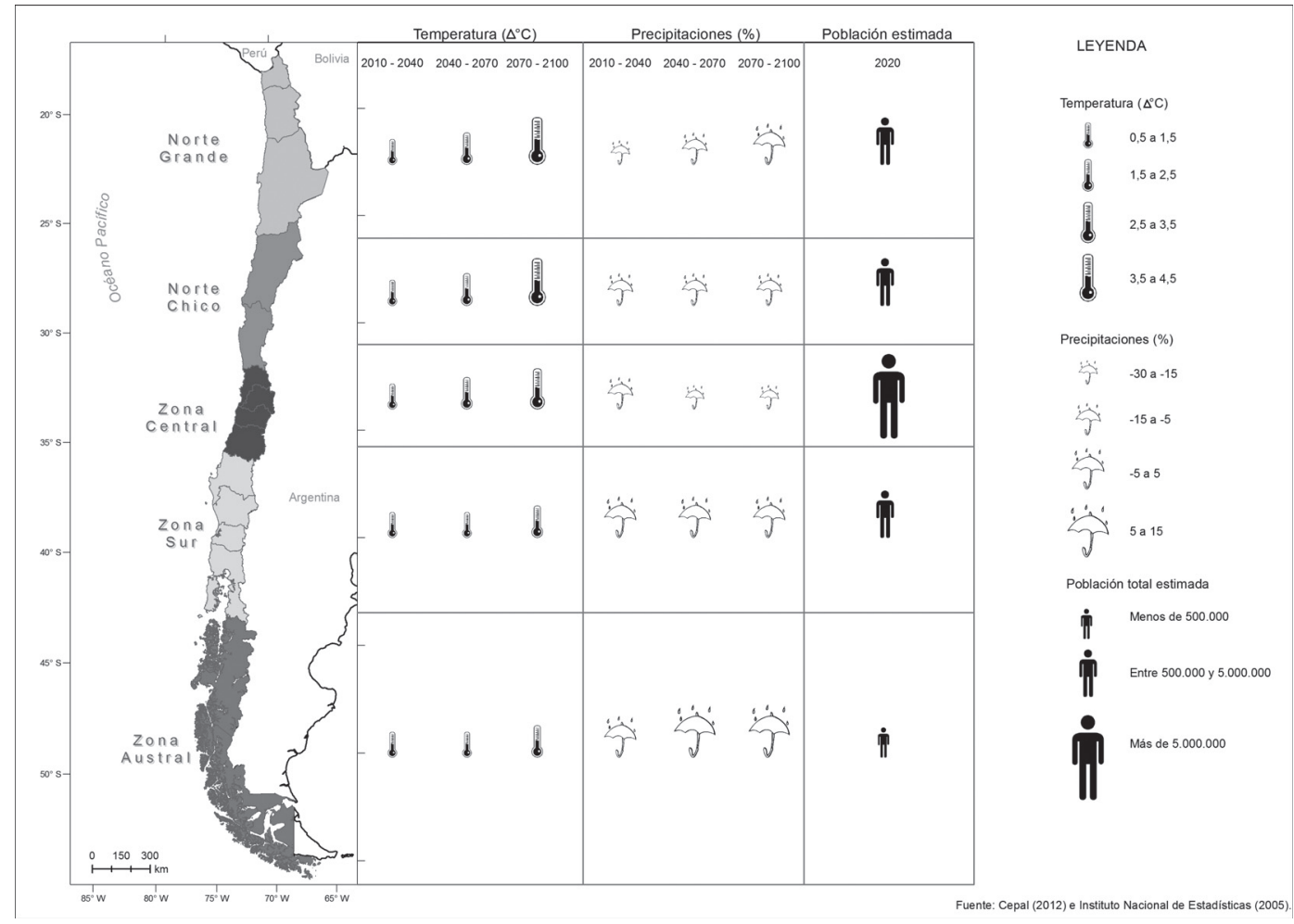

Fuente: Elaboración propia con datos de CEPAL (2012) e INE (2015).

De todas formas los impactos más latentes se verían en el Norte Chico y Zona Central, donde se estima una disminución de las precipitaciones y un aumento de las temperaturas, lo que incidiría fuertemente en los desastres asociados a la sequía. Si bien no se ha registrado ninguna zona de catástrofe por olas de calor, es probable que a futuro este fenómeno vaya surgiendo especialmente en comunas ubicadas en los valles interiores.

Con respecto a la población proyectada para el año 2020, se observa que la macrozona Central tendrá la mayor cantidad de población y junto con ello una eventual mayor vulnerabilidad, explicado por la presencia de la metrópolis de Santiago, principal polo económico y demográfico del país, la conurbación de Valparaíso-Viña del Mar y otras ciudades intermedias.

\section{Revisión de comunas peor evaluadas}

Para mejorar la situación de las comunas peor evaluadas mediante el índice de riesgo climático aplicado, la gestión del riesgo debiese enfocarse en aquellos aspectos más débiles tanto en temas de vulnerabilidad como de resiliencia. Por ejemplo, la comuna de La Ligua debiese enfocar su gestión en mejorar su sistema de salud ya que presenta un elevado porcentaje de población expuesta que presentó enfermedades respiratorias el año 2009 , esto es $2,2 \%$, cifra que supera la media nacional. Este dato es relevante dada la cantidad y tipología de amenazas climáticas, como por ejemplo heladas y temporales. No obstante, es importante mencionar que esta comuna logró hace poco (2013) la aproba- 
ción y financiamiento de un proyecto de Centro de Salud Familiar (CESFAM) para 30.000 habitantes, iniciativa muy positiva para la reducción de su vulnerabilidad y de las comunas vecinas, ya que de esta manera se puede descongestionar la demanda sobre el hospital comunal San Agustín, que no da abasto para la población, especialmente en la estación de invierno, donde el hospital generalmente colapsa debido a la falta de espacios para los enfermos (Díaz, 2012).

Sin embargo, la reducción de vulnerabilidad de la comuna de La Ligua no solo debe estar enfocada en el mejoramiento de la salud pública, puesto que también presenta cifras desfavorables en participación en organizaciones sociales, ya que se queda atrás respecto a las otras comunas de la provincia con un $23 \%$, cifra inferior al promedio nacional de $25 \%$. La participación de la población en este tipo de organizaciones es muy relevante para las fases de prevención, emergencia y recuperación ante un evento climático extremo. Mejorar este aspecto es clave para que la comuna aumente su resiliencia y disminuya su vulnerabilidad ante eventos de manifestación inmediata o de largo plazo. Como referencia se puede indicar que el nivel de participación en organizaciones de nivel comunal en países como Brasil asciende a 38,3\% (Bizberg, 2010).

Por otra parte, la comuna de Petorca es la más vulnerable en temas de pobreza, con un $16,8 \%$ de población en situación de pobreza al año 2009, esta situación podría ser explicada por sus bajos niveles de ingreso (US\$ 625 promedio mensual de ingreso autónomo por hogar a nivel comunal versus un promedio de US\$1.164 de ingreso autónomo en el país) y sus altos niveles de analfabetización (8\%) comparados con la realidad nacional (3,9\%) (BCN, 2013). A pesar de esta desfavorable situación, la comuna de Petorca presenta un alto porcentaje de participación en organizaciones sociales $(37,2 \%)$, lo que si bien en términos del índice de riesgo ayuda a aumentar la resiliencia, no compensa el mal desempeño en las otras dimensiones. Por su parte, la comuna de Cabildo también tiene un alto porcentaje comparativo de participación en organizaciones sociales $(29,7 \%)$, pero presenta el índice más bajo de desarrollo humano de las comunas analizadas $(0,671)$, por lo que la reducción de la vulnerabilidad en esta comuna debe estar enfocada en el mejoramiento integral de las cifras comunales.

Es importante señalar que las comunas de la provincia de Petorca: La Ligua, Cabildo y Petorca, son comunas con una marcada vocación agrícola. El $85,5 \%$ de la superficie de Petorca es cultivada, $54,6 \%$ en Cabildo y $31,4 \%$ en La Ligua (INE, 2008). Uno de los principales cultivos es la palta con el $90 \%$ de su superficie destinada a este cultivo. Estas comunas son muy sensibles a los impactos del clima por la sobreexplotación del recurso agua y la asignación de derechos de aprovechamiento de aguas más allá de la capacidad física de la cuenca y de los acuíferos. La sequía que ha afectado a esta provincia "ha provocado la pauperización de más de 7.000 pequeños agricultores, más de 50.000 personas padecen de la carencia de agua para beber y servicios higiénicos básicos, a pesar de las innumerables denuncias realizadas" (Instituto Nacional de Derechos Humanos, 2010: 142). En este caso, la gestión debería apuntar a lo que O'Hare y Rivas (2005) describen como medidas de adaptación soft, o no estructurales, en donde los recursos se enfocan en acciones para reducir la vulnerabilidad de la población en el corto y mediano plazo.

Fuera del ámbito del índice aplicado, el año 2013 la macrozona Central del país fue testigo de los problemas derivados de intensas lluvias estivales durante el mes de enero y febrero que sumadas a las altas temperaturas y deshielos propios de la temporada de verano, generaron aluviones en la zona cordillerana de la ciudad de Santiago lo cual provocó el colapso masivo del servicio de agua potable en 22 comunas del AMS, produciéndose cortes del suministro (Diario El Mercurio, 2013). Esto es especialmente relevante, puesto que no todos los eventos extremos desecandenan una declaratoria de zona de catástrofe, sin embargo estos eventos pueden ocasionar pérdidas y perjuicios tanto como los ligados a la zonas de catátrofes y por lo tanto, deben enfrentarse de una manera más preventiva que reactiva.

Finalmente, cabe destacar que hay cierto tipo de riesgos que son de manifestación más lenta, como por ejemplo las sequías o el proceso de desertificación. Desde el año 
2007 se observa una escasa cantidad de precipitación total anual para la zona central de Chile, determinando que la década 20002010 es una de las más secas de los últimos 60 años, siendo el año 2002, el último año Iluvioso observado (Dirección Meteorológica de Chile, 2013).

\section{Consideraciones finales}

En relación al índice de riesgo climático propuesto se puede concluir que no necesariamente las comunas con mayor nivel socioeconómico tienen mejores niveles de resiliencia. Esto es algo que debe resaltarse, ya que es muy importante contar con una comunidad comprometida y organizada que fortalezcan la capacidad de respuesta ante eventos extremos.

Por otro lado, es importante tener presente la importancia de espacializar el riesgo climático para poder focalizar eficientemente los recursos y las políticas públicas.

Del mismo modo, existen varias indicaciones para mejorar el índice propuesto: en primer lugar se debe considerar que el indicador de amenaza está basado en una visión retrospectiva de las zonas de catástrofes y no en las dinámicas naturales propiamente tales; al mismo tiempo según la base de datos consultada no fue posible acceder a las declaraciones de zonas de catástrofes anteriores al año 1984; se pueden incluir otras fuentes de información, actualizadas y del mismo período de tiempo; o bien incluir nuevos indicadores que sean relevantes para la toma de decisiones, como por ejemplo las capacidades de gestión instaladas. Se enfatiza la importancia de seguir realizando este tipo de estudios a distintas escalas geográficas, que permitan evaluar el panorama actual y futuro para poder proponer medidas de adaptación adecuadas y anticiparse a crisis socionaturales.

En términos humanos, económicos y políticos resulta mucho más lógico aplicar medidas de adaptación climática en lugar que medidas reactivas como la declaración de zonas de catástrofe, que solo están dirigidas a atender la emergencia y no abordar toda la complejidad del problema. Por tal razón, otra conclusión tiene que ver con la necesidad de mejorar la política pública que enfrente los desafíos del cambio climático, pasando por mejorar nuestros instrumentos de planificación territorial y de coordinación de emergencias como también la materialización de obras de infraestructura que permitan disminuir los niveles de vulnerabilidad y aumentar los de resiliencia. Infraestructura para enfrentar la sequía, diseño de obras hidraúlicas para periodos de retornos mayores, planificación de áreas verdes que actúen como buffers frente a amenazas hidrometeorológicas son algunos ejemplos que se pueden implementar para prepararse ante eventos extremos.

Finalmente, es importante centrar los esfuerzos en aquellas regiones que se verán a futuro mayormente afectadas por los impactos del cambio climático y variabilidad natural del clima, de manera de cambiar el enfoque reactivo, como es la declaratoria de zona de desastres, por un enfoque proactivo y estratégico como la gestión efectiva del riesgo de desastre. Las regiones y comunas más vulnerables y riesgosas frente al cambio climático corresponden a la zona de Chile central, especialmente en la costa y valles interiores, donde se concentrará mayormente la población y se pronostica una agudización de los problemas climáticos e hidrometeorológicos observados como sequías, inundaciones, heladas, así como marejadas, incendios, olas de calor, entre otros y que requieren de una mayor preparación.

\section{Referencias bibliográficas}

ARENAS, F.; HIDALGO, R. y LAGOS, M. Los riesgos naturales en la planificacion territorial. Santiago de Chile: Centro de Políticas Públicas UC, 2010.

AYALA-CARCEDO, F.J. y OLCINA, J. Riesgos Naturales. Barcelona: Editorial Ariel, 2002.

BIBLIOTECA DEL CONGRESO NACIONAL DE CHILE (BCN). Reportes Estadísticos y Comunales, 2013. Disponible en Internet: http:// reportescomunales.bcn.cl/ 
BIBLIOTECA DEL CONGRESO NACIONAL DE CHILE (BCN). Base de datos de Leyes chilenas. Disponible en Internet: http://www.leychile. $\mathrm{cl} /$

BIZBERG, I. Una Democracia Vacía. Sociedad Civil, Movimientos Sociales y Democracia. En: BIZBERG, I. y ZAPATA, F. (coordinadores). Movimientos sociales. México: El Colegio de México, 2010.

CARVACHO, L. Asigcon, propuesta de un método para la comparación de unidades espaciales utilizando variables normalizadas sobre una base conceptual. Anales del XXXII Congreso Nacional y XVII Internacional de Geografía: Conciencia Geográfica en el Tercer Milenio, 2012, p. 448-455.

CEPAL. La Economía del Cambio Climático en Chile. Síntesis. Santiago de Chile: Comisión Económica para América Latina, Naciones Unidas, 2012.

CONSTITUCIÓN POLÍTICA DE LA REPÚBLICA DE CHILE 1980. Ministerio Secretaría General de la Presidencia. Diario Oficial de la República de Chile. Santiago de Chile, 17 de septiembre de 2005 (Decreto 100).

D'AMATO, G.; CECCHI, L.; D'AMATO, $M$. \& ANNESI-MAESANO, I. Climate change and respiratory diseases. European Respiratory Review, 2014, No 23, p. 161-169.

DECRETO SUPREMO No 104. Chile. Fija el texto refundido, coordinado y sistematizado del Título I de la Ley $N^{\circ}$ 16.282. Diario Oficial de la República de Chile, Santiago de Chile, 25 de junio de 1977.

DEIS. Base de datos egresos hospitalarios 2001-2012. Santiago de Chile: Departamento de Estadísticas e Información de Salud, Ministerio de Salud [base de datos], 2012.

DEPARTAMENTO DE GEOFÍSICA DE LA UNIVERSIDAD DE CHILE/ Comisión Nacional del Medio Ambiente (DGF/ CONAMA). Estudio de la variabilidad climática en Chile para el siglo XXI. Santiago de Chile, 2006.

DIARIO EL MERCURIO. Aguas Andinas compensará con hasta $\$ 4.000$ a clientes afectados por cortes de agua en la RM. Santiago de Chile: Diario El Mercurio, Sección Economía, 2013. Disponible en Internet:

http://www.emol.com

DÍAZ, J. Aprueban recursos para diseño de construcción de centro de salud familiar de La Ligua. La Ligua: Ilustre Municipalidad de La Ligua, 2012. Disponible en Internet: http://www. laligua.cl/noti01.php?id=437

DIRECCIÓN METEOROLÓGICA DE CHILE. Boletín N¹ Monitoreo de Sequía Meteorológica. Santiago de Chile, 2013.

FALVEY, M. \& GARREAUD, R. Regional cooling in a warming world: Recent temperature trends in the southeast Pacific and along the west coast of subtropical South America (1979-2006). Journal of Geophysical Research Atmospheres, 2009, Vol. 114, D04102, doi:10.1029/2008JD010519

GALLOPÍN, G. Linkages between vulnerability, resilience, and adaptative capacity. Global Environmental Change, 2006, 16, p. 293-303.

INSTITUTO NACIONAL DE DERECHOS HUMANOS (INDH). Mapa de Conflictos Socioambientales en Chile. Santiago de Chile: Unidad de Estudio, INDH, 2012.

INSTITUTO NACIONAL DE ESTADÍSTICAS (INE). Censo de Población y Vivienda 2002. Santiago de Chile: INE, 2002.

INSTITUTO NACIONAL DE ESTADÍSTICAS (INE). Chile: proyecciones y estimaciones de población. 1990-2020. Santiago de Chile: INE, 2005.

INSTITUTO NACIONAL DE ESTADÍSTICAS (INE). VII Censo Nacional Agropecuario año agrícola 2006-2007. Santiago de Chile: INE, 2008.

INTERGOVERNMENTAL PANEL ON CLIMATE CHANGE (IPCC). Managing the Risks of Extreme Events and Disasters to Advance Climate Change Adaptation. Special Report of the Intergovernmental Panel on Climate Change. New York: Cambridge University Press, 2012.

INTERGOVERNMENTAL PANEL ON CLIMATE CHANGE (IPCC). Climate Change 2014: Impacts, Adaptation, and Vulnerability. Part A: 
Global and sectoral Aspects. Working Group II Contribution to the Fifth Assessment Report of the Intergovernmental Panel on Climate Change. Cambridge/New York: Cambridge University Press, 2014.

KLEIN, R.J.; SMITH, M.J.; GOOSEN, H. HULSBERGEN, C.H. Resilience and Vulnerability: Coastal Dynamics or Dutch Dikes? The Geographical Journal, 1998, Vol. 164, № 3, p. 259-268.

LEY $N^{\circ}$ 16.282. Chile. Fija Disposiciones para Casos de Sismos o Catástrofes. Establece Normas para la Reconstruccion de la Zona Afectada por el Sismo de 28 de Marzo de 1965 y Modifica la Ley $N^{\circ}$ 16.250. Ministerio de Hacienda. Diario Oficial de la República de Chile, Santiago de Chile, 28 de julio de 1965.

LEY N 18.415. Chile. Ley Orgánica Constitucional de los Estados de Excepción. Ministerio del Interior. Diario Oficial de la República de Chile, Santiago de Chile, 14 de junio de 1985.

MINISTERIO DE DESARROLLO SOCIAL. Incidencia de la Pobreza a nivel Comunal, según Metodología de Estimación para Áreas Pequeñas. Chile 2009 y 2011. Santiago de Chile: Observatorio Social, Serie Informes Comunales $N^{0} 1,6$ de febrero de 2013.

MINISTERIO DE PLANIFICACIÓN Y COOPERACIÓN (MIDEPLAN). Encuesta de caracterización socioeconómica nacional CASEN 2009. Módulo comunal [Base de datos]. Santiago de Chile: Departamento de Información Social, División Social, 2009.

ORGANIZACIÓN PARA LA COOPERACIÓN Y EL DESARROLLO ECONÓMICO (OCDE). Society at a Glance 2014: OECD: Social Indicators. OECD Publishing, 2014. Disponible en Internet:

http://dx.doi.org/10.1787/soc_glance-2014-en

OFICINA NACIONAL DE EMERGENCIAS (ONEMI). Web institucional. Disponible en Internet: http://www.onemi.cl/

OFICINA NACIONAL DE EMERGENCIA (ONEMI). Informe consolidado temporales 1 junio - 2 de julio 2000. Santiago de Chile: Departamento de Protección Civil, ONEMI, 2000.
OFICINA NACIONAL DE EMERGENCIA (ONEMI). Política Nacional para la Gestión de Riesgo de Desastres. Santiago de Chile: ONEMI, 2014.

O'HARE, G. \& RIVAS, S. The landslide hazard and human vulnerability in La Paz, Bolivia. The Geographical Journal, 2005, Vol. 171, № 3 , p. 239-258.

MINISTERIO DE PLANIFICACIÓN Y COOPERACIÓN (MIDEPLAN) y PROGRAMA DE LAS NACIONES UNIDAS PARA EL DESARROLLO (PNUD). Las trayectorias del desarrollo humano en las comunas de Chile, 1994-2003. Santiago de Chile: División Social del Ministerio de Planificación y Cooperación (MIDEPLAN) y Equipo de Desarrollo Humano del Programa de las Naciones Unidas para el Desarrollo (PNUD), 2005.

ECHO. Documento País. Análisis de riesgos de desastres en Chile. VII Plan de Acción DIPECHO en Sudamérica 2011-2012. Santiago de Chile: Organización de las Naciones Unidas para la Educación la Ciencia y la Cultura (UNESCO), PNUD, Cruz Roja Chilena y DG ECHO, 2012.

UNISDR. Terminología sobre Reducción del Riesgo de Desastres. Estrategia Internacional para la Reducción de Riesgos de las Naciones Unidas (UNISDR). Disponible en Internet: http://www.unisdr.org/les/7817_UNISDRTerminologySpanish.pdf.

VILLARROEL, C. Eventos extremos de precipitación y temperatura en Chile: proyecciones para fines del siglo XXI. Santiago de Chile: Tesis (Magister en Meteorología y Climatología), Universidad de Chile, Facultad de Ciencias Físicas y Matemáticas, Departamento de Geofísica, 2013.

WALKER, B.; CARPENTER, S.; ANDERIES, J.; ABEL, N.; CUMMING, G.; JANSSEN, M.; LEBEL, L.; NORBERG, J.; PETERSON, G.D. \& PRITCHARD, R. Resilience Management in Social-ecological Systems: a Working Hypothesis for a Participatory Approach. Ecology and society, 2002, Vol. 6, № 1, Art. 14. Disponible en Internet:

http://www.consecol.org/vol6/iss1/art14/

WILCHES-CHAUX, G. Herramientas para la Crisis: Desastres, Ecologismo y Formación Profesional. Popayán: Servicio Nacional de Aprendizaje (SENA), 1989. 
